\title{
Farman Institute 3D Point Sets - High Precision 3D Data Sets
}

\author{
Julie Digne $^{1}$, Nicolas Audfray ${ }^{2}$, Claire Lartigue ${ }^{2}$, Charyar Mehdi-Souzani $^{2}$, \\ Jean-Michel Morel ${ }^{1}$ \\ ${ }^{1}$ CMLA, ENS Cachan, France \\ (\{julie.digne, morel\}@cmla.ens-cachan.fr) \\ ${ }^{2}$ LURPA, ENS Cachan, France \\ (\{nicolas.audfray, claire.lartigue, charyar.mehdi-souzani\}@lurpa.ens-cachan.fr)
}

Communicated by Jose-Luis Lisani

\begin{abstract}
This article is dedicated to describe a new type of data: high precision raw data coming from the acquisition of objects by a $3 \mathrm{D}$ laser scanner.
\end{abstract}

\section{Supplementary Material}

The datasets described in this article can be downloaded from the associated IPOL web page ${ }^{1}$.

Keywords: 3D; datasets; point sets

\section{Introduction}

This article is dedicated to describe a new type of data: high precision raw data coming from the acquisition of objects by a 3D laser scanner. The obtained datasets consist of unoriented and non triangulated 3D point clouds, which can be further processed by the techniques described in [1, 2, 3].

The article is organized as follows: Section 2 reviews the data acquisition process; the file format used to store the acquired data is described in Section 3; the datasets available for download are shown in Section 4; sections 5 and 6 are devoted to explain how the stored data can be read from a file and visualized. 


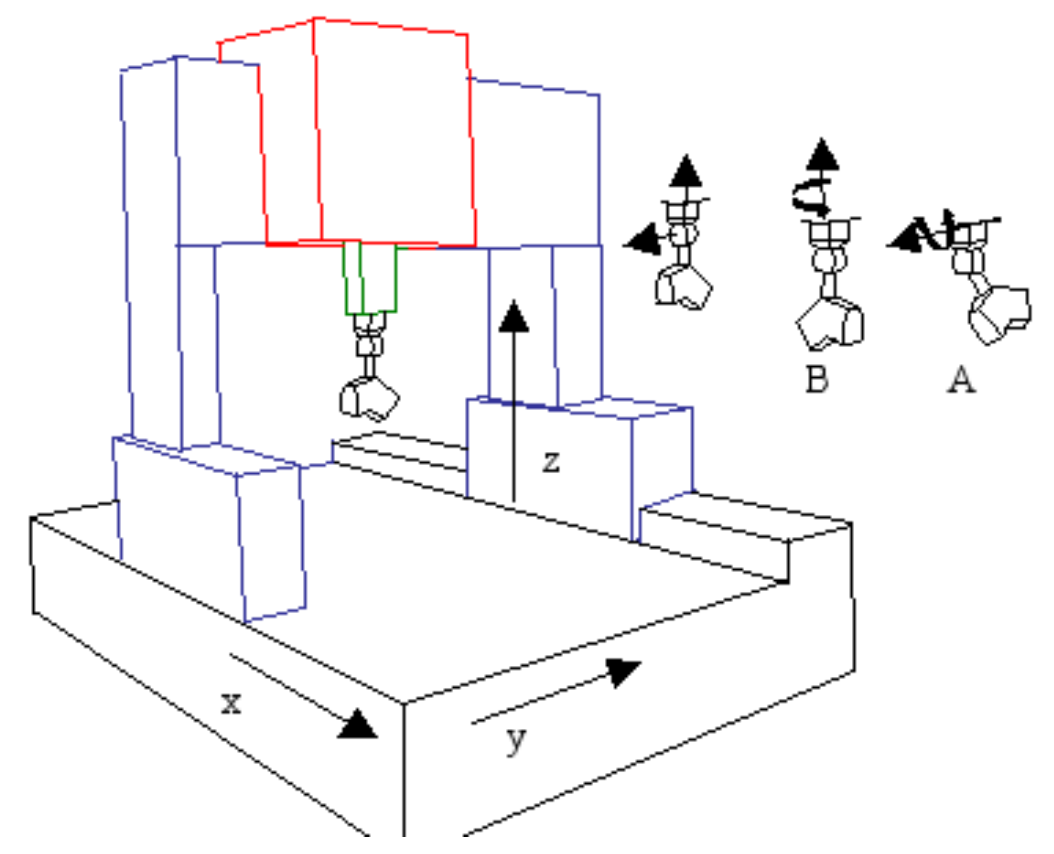

Figure 1: Scheme of the Acquisition System.

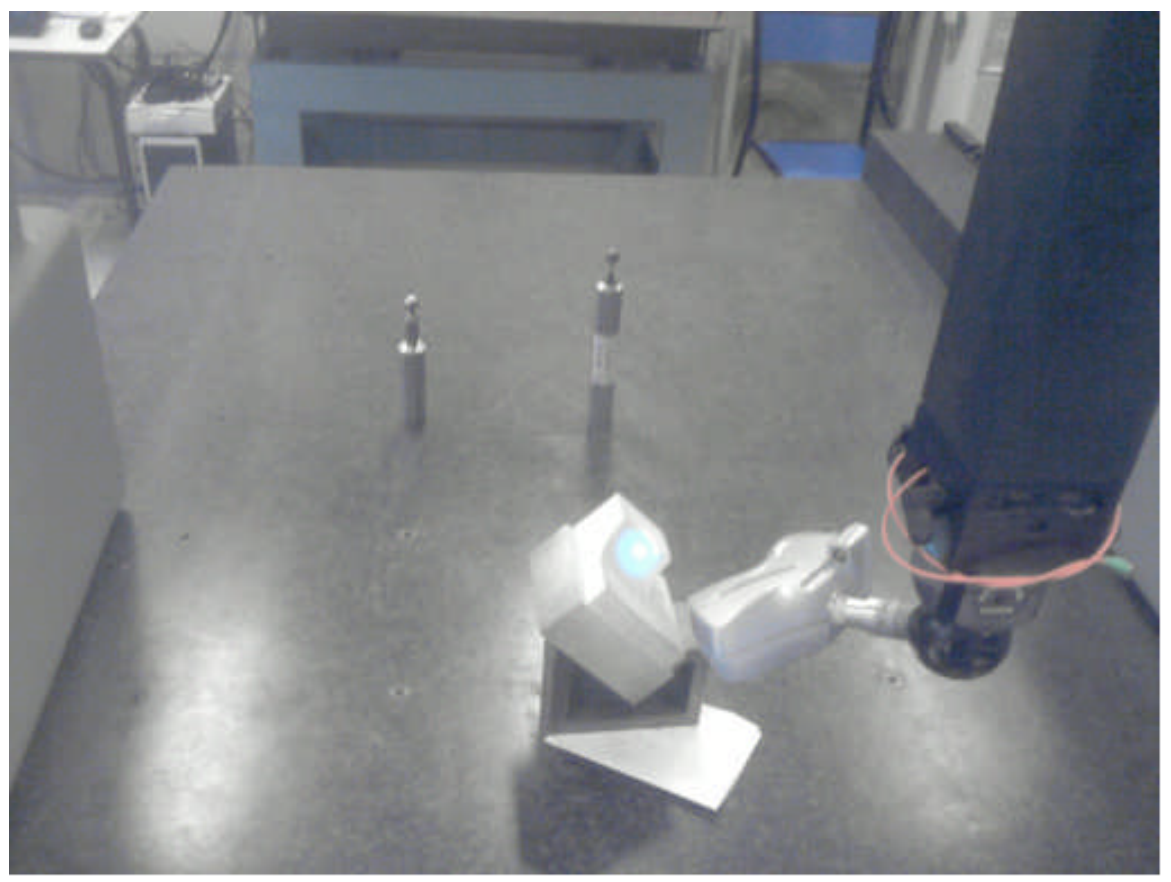

Figure 2: Laser ray projected on the surface of the object to digitize.

\section{Acquisition Process}

The experimental scanning device is made of three distinct parts: a displacement system, a digitizing sensor and a data processing system.

The displacement system is a Coordinate Measuring Machine (CMM) headed with a contactfree laser-plane sensor (the digitizing system). This scanner is connected to the CMM through a motorized indexing head as shown in Figures 1 and 2. The two scanner head orientations are given by two angles $\mathrm{A}$ and $\mathrm{B}$ that can only be incremented by $7.5^{\circ}$.

\footnotetext{
${ }^{1}$ https://doi.org/10.5201/ipol.2011.dalmm_ps
} 
The acquisition system is based on the geometric principle of triangulation. A laser source emits a laser ray diffracted to a laser plane, and a CCD camera captures the intersection line between the laser plane and the surface of the object [4]. Since the angle between the camera and the laser ray emitter is fixed, the position of the acquired laser ray in the CCD matrix yields the 3D coordinates in the sensor coordinate system. As the position and the orientation of the scanner is known in the CMM frame, by using the calibration model of the whole system, the coordinates can be expressed in the CMM coordinate system.

\subsection{Acquisition}

The digital acquisition of an object needs generally several different sensor orientations. In order for a point to be acquired, it must be hit by the laser ray and seen by the CCD camera at the same time. The three translations of the CMM and the two rotations of the head allow for the high quality acquisition of freeform shapes, without repositioning the object. For each orientation, the sensor has to be calibrated on a reference measurement artifact, in this case a perfect sphere. The diameter and the position of the sphere are known with an uncertainty inferior to $0.001 \mathrm{~mm}$. The center of this sphere is the origin of the CMM coordinate system. The calibration operation consists in scanning the sphere in different areas of the CCD camera [5]. As we perfectly know the size and the location of the sphere, all calibration parameters are computed for each sensor orientation. This calibration step reduces errors due to optical measurement parts of the scanner, and allows for the definition of all the objects directly in the same coordinate system. However, no digitization system is perfect. Some calibration and registration errors remain after calibration. It is nevertheless still possible to increase the quality of digitized point clouds. The distance between the captor and the object, the acquisition angle and the sensor reorientation are crucial to generate a high quality point cloud. In [5] it is shown how the error varies with respect to these settings. The result is also very dependent on the physical nature of the surface (reflectance, specularity). For some reflecting objects a white powder has to be spread on the surface before the digitization (see the Mire dataset for example).

A single sweep using a given sensor orientation is hardly enough to cover the whole surface of the object. This is due to the width of the laser ray, each sweep covers a band of the surface. Therefore, one has to do several sweeps with the same orientation only modifying the trajectory of the sensor so that it covers the whole surface.

\subsection{Equipment Details}

We use as captor a Zephyr KZ25 manufactured by Kreon technologies. Both frequency and power of the laser ray can be set depending on the surface to probe. A Look Up Table allows for setting an intensity threshold above which a trace appearing on the CCD camera should be taken into account. The settings aim at ensuring a trace width between 7 and 9 micrometers: the laser power is set to $4 \mathrm{mV}$ and the integration time is $1 / 250 \mathrm{~s}$.

This captor is controlled by a rotating head. This rotating head is a Renishaw PH10M with a repeatability of 0.38 micrometers (constructor value).

The sphere used for calibration is manufactured and certified by Kreon Technologies. During the calibration stage, the difference between the computed diameter and the theoretical diameter $(15,993 \mathrm{~mm})$ is used as well as the deviation of the points relatively to a perfect sphere. 


\section{3D Data File Format}

Each data set is given as a set of compressed ASCII files (tgz). Each file represents a single sweep in a given scanning orientation. When more than one file is provided for a single orientation, each of the corresponding files is given a number. Each file is named Ax-By_n.asc where $x$ is the first orientation angle, $y$ is the second orientation angle and $n$ is the number of the sweep. The data sets are raw data point sets, in the sense that no preprocessing was applied, it is the direct output of the acquisition system.

For example, consider the Dame de Brassempouy dataset. It consists of 18 files: A45_B0.asc, A45_B0_2 asc, A45_B0_3. asc, A45_B-22 , 5.asc, A45_B-22 ,5_2.asc, A90_B-180.asc, A90_B-180_2 asc, A90_B-180_3.asc, A90_B-90_2.asc, A90_B-90_3. asc, A90_B-90_4.asc, A90_B90.asc, A90_B90_2 asc, A90_B90_3.asc, A90_B90_4.asc, A90_B-135.asc, A90_B-135_2.asc, A90_B-135_3.asc.

The object was acquired using 6 different orientations that can be read in the names of the files: $(45,0),(45,-22.5),(90,-180),(90,-90),(90,90),(90,-135) .18$ sweeps were required (corresponding to the 18 files).

Each file contains $N$ lines (one line per point) with the point coordinates in a single row. Notice that there is no normal information.

For example, we list the 10 first lines of file A45_B0 .asc, still in the Dame de Brassempouy dataset:

$$
\begin{array}{lll}
-524.21393 & 4.72374 & -31.67768 \\
-524.22937 & 4.72252 & -31.76848 \\
-524.22412 & 4.72135 & -31.83910 \\
-524.18768 & 4.72026 & -31.87937 \\
-524.16681 & 4.71914 & -31.93484 \\
-524.13818 & 4.71803 & -31.98271 \\
-524.09912 & 4.71696 & -32.02045 \\
-524.07050 & 4.71585 & -32.06833 \\
-524.04181 & 4.71475 & -32.11621 \\
-523.98969 & 4.71253 & -32.21708
\end{array}
$$

Each row corresponds to the $\mathrm{x}, \mathrm{y}, \mathrm{z}$ coordinates of a point of the first sweep with orientation $(45,0)$. Coordinates are given in millimeters. The whole system is calibrated so that all coordinates are given in the same coordinate system.

A set of pictures of the data sets is also provided in a downloadable tar.gz archive. The physical size of each object (height $\times$ width $\times$ depth with respect to the provided picture), the number of sweeps and the total point number are also given.

\section{Data}

The objects presented here are reproductions bought from Le Louvre Museum, Paris, France (http: //www. boutiquesdemusees.fr/en/), with the exception of the Mask and Tanagra which come from the Museum of Cycladic Arts (Athens, Greece). Most of the objects are made of resin.

\subsection{Tanagra}

- Object size: $22 \times 7.5 \times 9 \mathrm{~cm}$

- Description: 160 sweeps, 17,496,999 points, 186MBytes (see Figure 3)

- Data set: http://www.ipol.im/data/algo/d_point_cloud_data/farman_tanagra.tar.gz 
- High-resolution photographs: http://www.ipol.im/data/algo/d_point_cloud_data/farman_ tanagra_highres.tar.gz

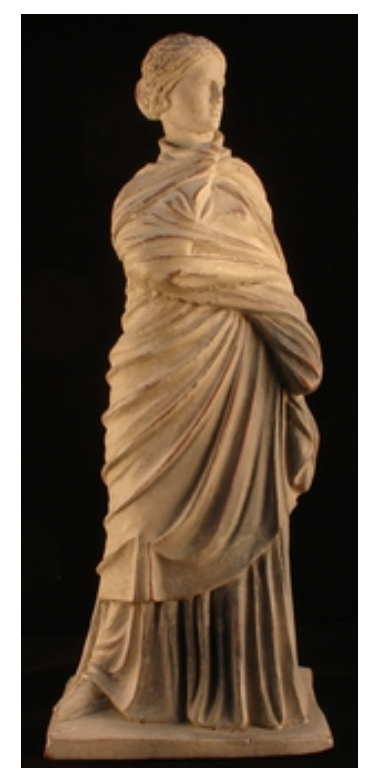

Figure 3: Tanagra image.

\subsection{Dancer with Crotales}

- Object size: $20 \times 11 \times 6 \mathrm{~cm}$

- Description: 94 sweeps, 5,524,627 points, 60MBytes (see Figure 4)

- Data set: http://www.ipol.im/data/algo/d_point_cloud_data/farman_crotales.tar.gz

- High-resolution photographs: http://www.ipol.im/data/algo/d_point_cloud_data/farman_ crotales_highres.tar.gz

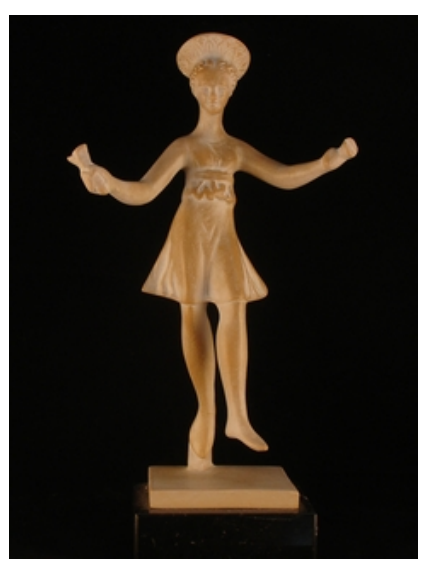

Figure 4: Dancer with Crotales image. 


\subsection{Mime}

- Object size: $17.5 \times 7 \times 5 \mathrm{~cm}$

- Description: 102 sweeps, 8,611,522 points, 93MBytes (see Figure 5)

- Data set: http://www.ipol.im/data/algo/d_point_cloud_data/farman_mime.tar.gz

- High-resolution photographs: http://www.ipol.im/data/algo/d_point_cloud_data/farman_ mime_highres.tar.gz

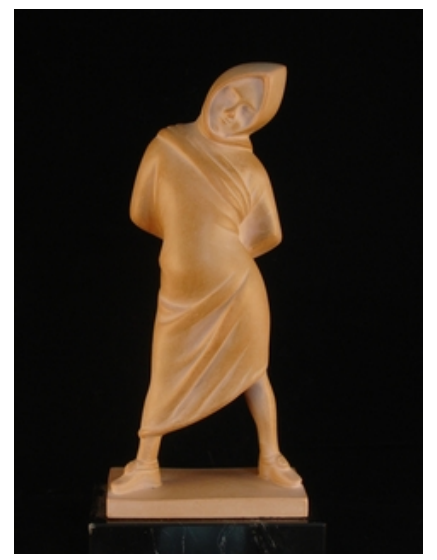

Figure 5: Mime image.

\subsection{Nefertiti}

- Object size: $19 \times 11 \times 6 \mathrm{~cm}$

- Description: 115 sweeps, 15,554,528 points, 168MBytes (see Figure 6)

- Data set: http://www.ipol.im/data/algo/d_point_cloud_data/farman_nefertiti.tar. gz

- High-resolution photographs: http://www.ipol.im/data/algo/d_point_cloud_data/farman_ nefertiti_highres.tar.gz

\subsection{Dame de Brassempouy}

- Object size: $6 \times 2 \times 2 \mathrm{~cm}$

- Description: 18 sweeps, 319,220 points, 3.5MBytes (see Figure 7)

- Data set: http://www.ipol.im/data/algo/d_point_cloud_data/farman_brassempouy.tar . gz

- High-resolution photographs: http://www.ipol.im/data/algo/d_point_cloud_data/farman_ brassempouy_highres.tar.gz 


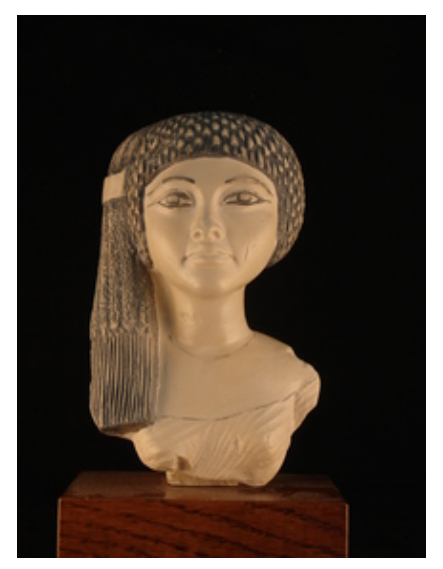

Figure 6: Nefertiti image.

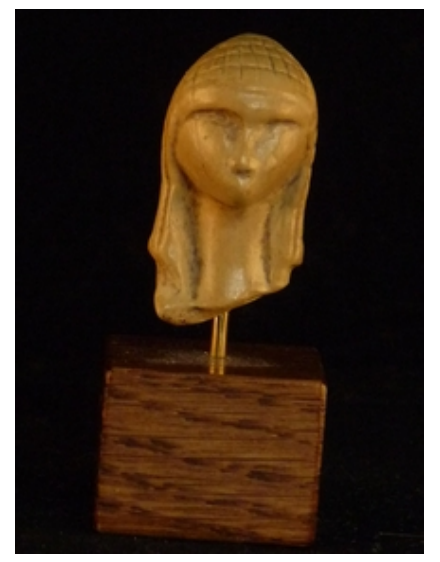

Figure 7: Dame de Brassempouy image.

\subsection{Pyramid}

- Object size: $8 \times 7.5 \times 7.5 \mathrm{~cm}$

- Description: 41 sweeps, 5,632,884 points, 59MBytes (see Figure 8)

- Data set: http://www.ipol.im/data/algo/d_point_cloud_data/farman_pyramid.tar.gz

- High-resolution photographs: http://www.ipol.im/data/algo/d_point_cloud_data/farman_ pyramid_highres.tar.gz

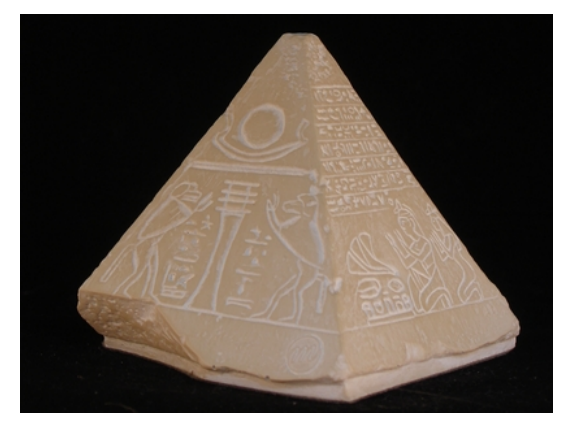

Figure 8: Pyramid image. 


\subsection{Mask}

- Object size: $16 \times 10 \times 6 \mathrm{~cm}$

- Description: 78 sweeps, 8,961,736 points, 99MBytes (see Figure 9)

- Data set: http://www.ipol.im/data/algo/d_point_cloud_data/farman_mask.tar.gz

- High-resolution photographs: http://www.ipol.im/data/algo/d_point_cloud_data/farman_ mask_highres.tar.gz

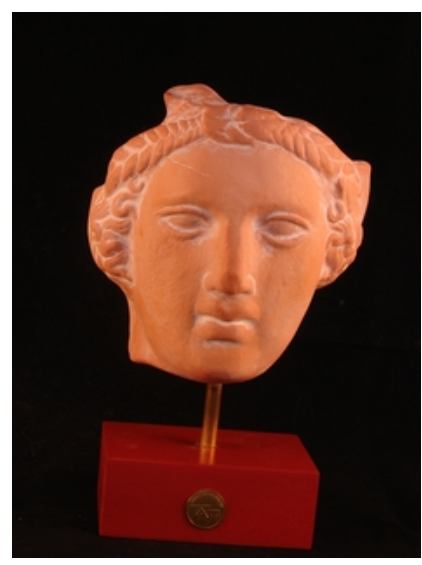

Figure 9: Mask image.

\subsection{Amants de Bordeaux}

- Object size: $8 \times 13 \times 7 \mathrm{~cm}$

- Description: 70 sweeps, 15,835,405 points, 168MBytes (see Figure 10)

- Data set: http://www.ipol.im/data/algo/d_point_cloud_data/farman_amants.tar.gz

- High-resolution photographs: http://www.ipol.im/data/algo/d_point_cloud_data/farman_ amants_highres.tar.gz

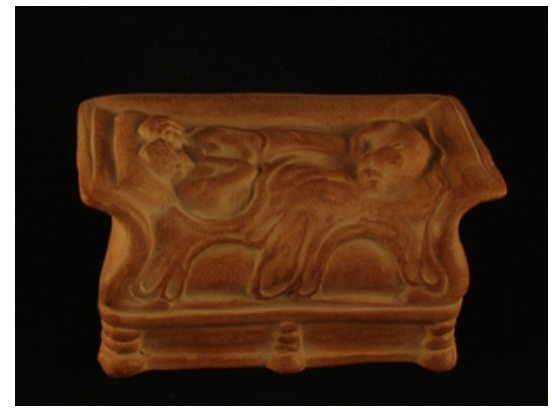

Figure 10: Amants de Bordeaux image. 


\subsection{Rosetta Stone}

- Object size: $32 \times 25.5 \times 1.5 \mathrm{~cm}$

- Description: 62 sweeps, 36,201,537 points, 363MBytes (see Figure 11)

- Data set: http://www.ipol.im/data/algo/d_point_cloud_data/farman_rosetta.tar.gz

- High-resolution photographs: http://www.ipol.im/data/algo/d_point_cloud_data/farman_ rosetta_highres.tar.gz

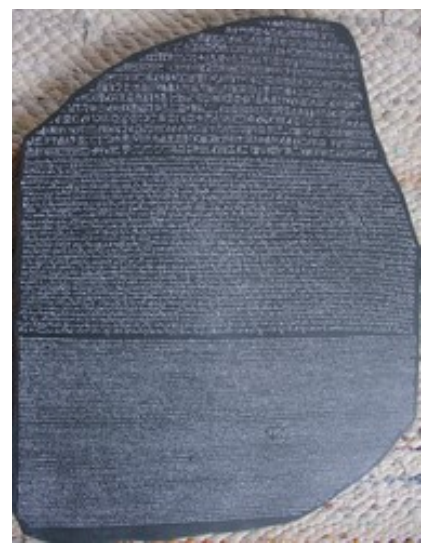

Figure 11: Rosetta Stone image.

\subsection{Anubis Bas-relief}

- Object size: $30 \times 20 \times 2 \mathrm{~cm}$

- Description: 13 sweeps, 9,991,040 points, 97MBytes (see Figure 12)

- Data set: http://www.ipol.im/data/algo/d_point_cloud_data/farman_anubis.tar.gz

- High-resolution photographs: http://www.ipol.im/data/algo/d_point_cloud_data/farman_ anubis_highres.tar.gz

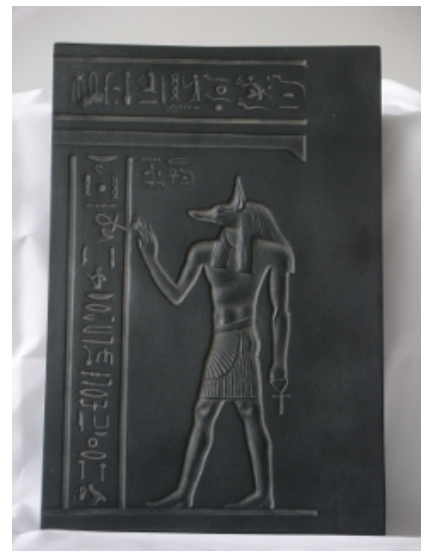

Figure 12: Anubis Bas-relief image. 


\subsection{Mire}

- Object size: $7 \times 15 \times 15 \mathrm{~cm}$

- Description: 75 sweeps, 16,111,522 points, 173MBytes (see Figure 13)

- Data set: http://www.ipol.im/data/algo/d_point_cloud_data/farman_mire.tar.gz

- High-resolution photographs: http://www.ipol.im/data/algo/d_point_cloud_data/farman_ mire_highres.tar.gz

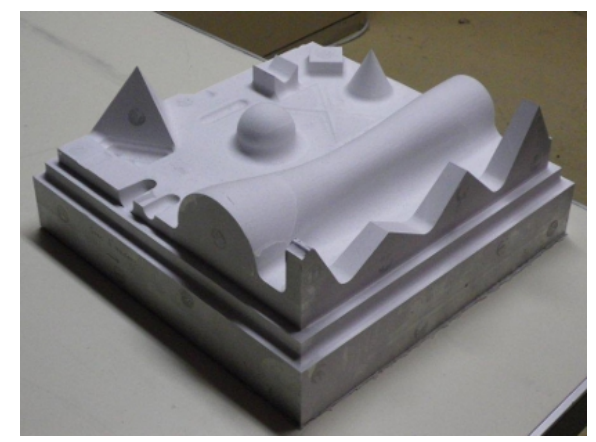

Figure 13: Mire image.

\section{Reading the Data}

The files are in plain ASCII. Reading them is straightforward from $\mathrm{C}$ or $\mathrm{C}++$ (or any other programming language). We provide an example of a $\mathrm{C}++$ program that reads points from a file "example.txt" and outputs all the points (one point per line) on the standard output.

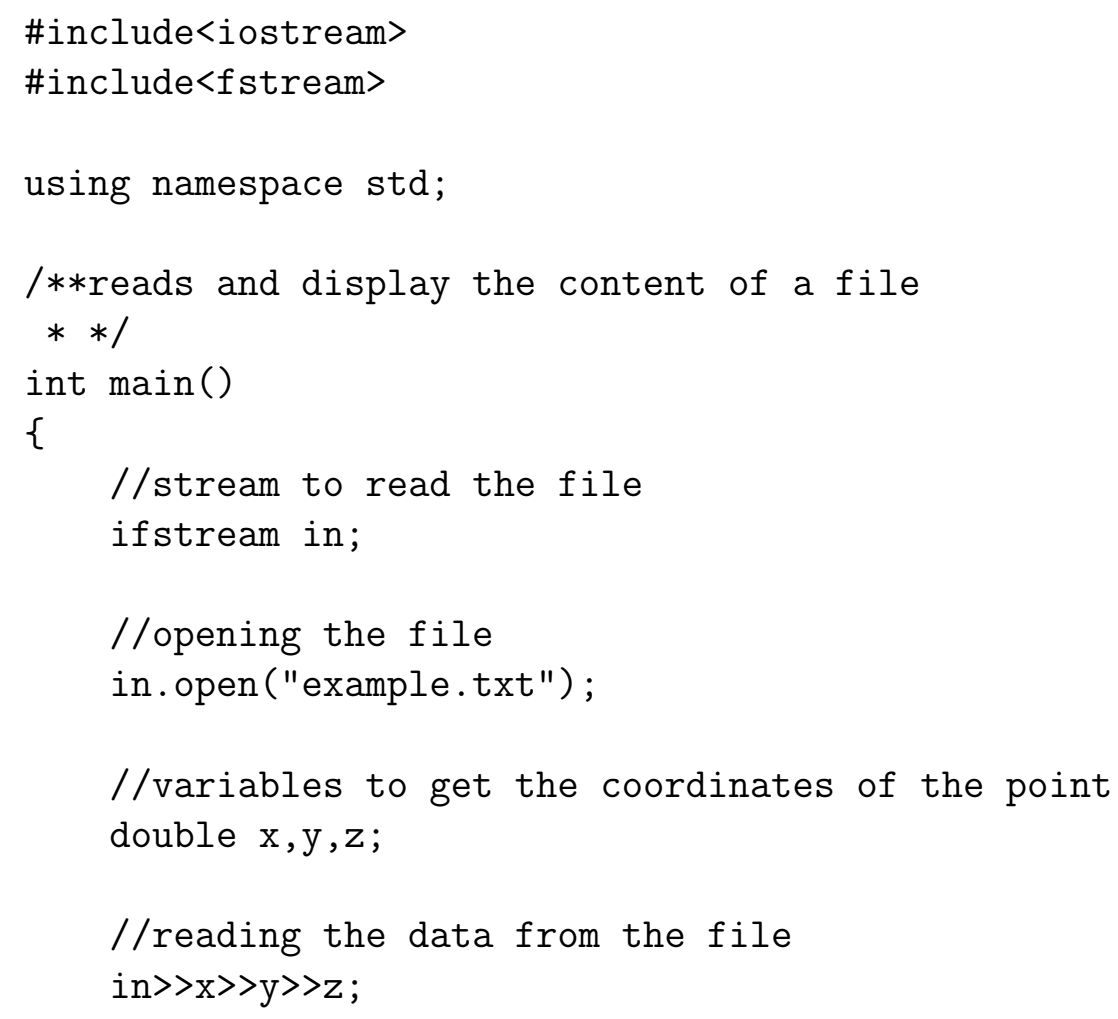




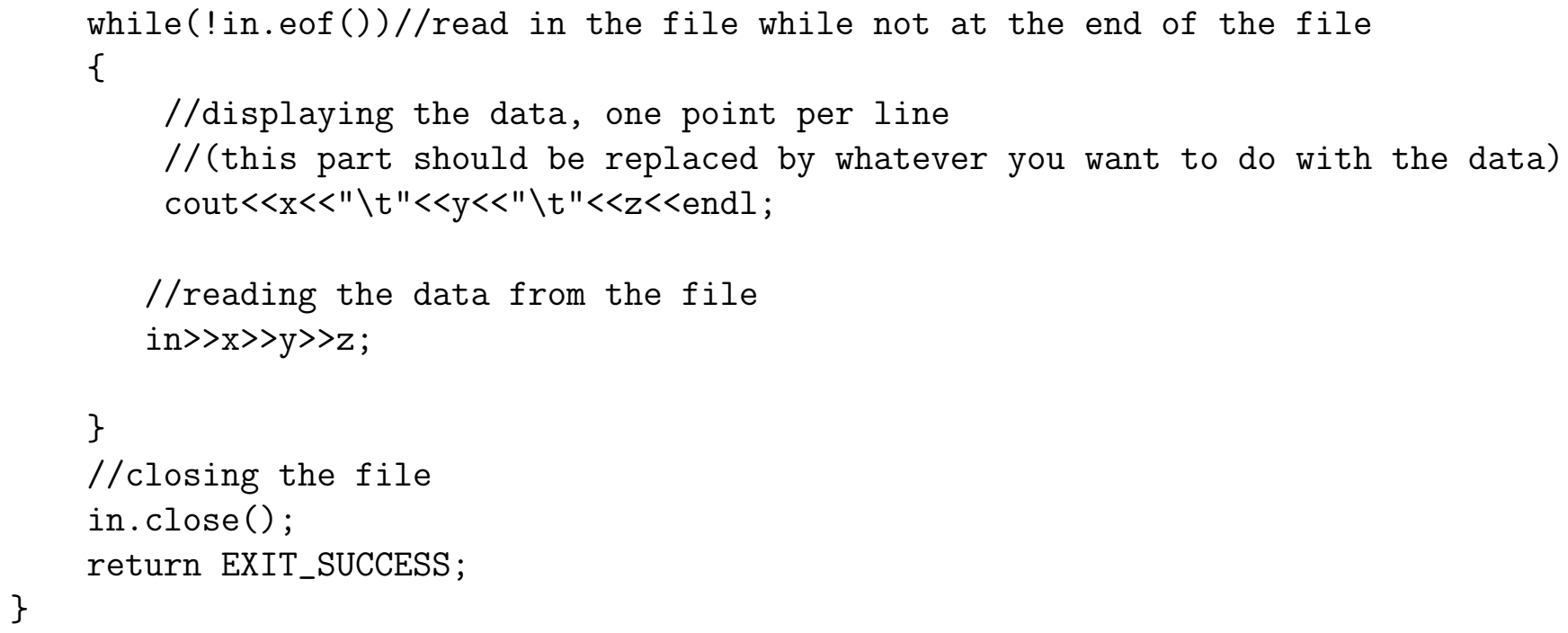

\section{Visualizing the Data}

Visualization of unoriented and non triangulated 3D point clouds is a difficult problem. In this case the clouds are so dense that it would result in visualizing the silhouette of the shape. However, if for some reason, the point cloud has to be visualized then the following MATLAB/Octave routine is sufficient:

$A=$ load ('data.txt');

$\operatorname{plot3}\left(A(:, 1), A(:, 2), A(:, 3),^{\prime} .^{\prime}\right)$; axis equal;

\section{Image Credits}

All images by the authors.

\section{References}

[1] J. Digne, J.-M. Morel, C. Mehdi-Souzani, C. Lartigue, "Scale Space Meshing of Raw Data Point Sets", Computer Graphics Forum, 2011. http://dx.doi.org/10.1111/j.1467-8659. $2011.01848 . \mathrm{x}$

[2] J. Digne, J.-M. Morel, N. Audfray, C. Lartigue, "High Fidelity Scan Merging", Computer Graphics Forum 29, no 5, pp 1643-1651, 2010. http://dx.doi.org/10.1111/j.1467-8659.2010. 01773.x

[3] C. Mehdi-Souzani, J. Digne, N. Audfray, C. Lartigue, J.-M. Morel, "Feature extraction from highdensity point clouds: toward automation of an intelligent 3D contact less digitizing strategy", CAD'10, Dubai June (21-25), 2010. http://dx.doi.org/10.3722/cadaps.2010.863-874

[4] C. Mehdi-Souzani, F. Thiebaut, C. Lartigue, "Scan planning strategy for a general digitized surface", Journal of Computing \& Information Science in Engineering, ASME International, Vol. 6, Issue 4, pp. 331-339, 2006. http://dx.doi.org/10.1115/1.2353853 
[5] C. Mehdi-Souzani, C. Lartigue, "Contact less laser-plane sensor assessment: toward a quality measurement", Proceedings of IDMME-Virtual Concept 2008, Beijing, China, 2008. 